\title{
Correlation between the occlusal vertical dimension and the pupil rima oris distance among sundanese population
}

\author{
Nur Emalina Akhma bt. Muhamad Shafree*, Taufik Sumarsongko*, Rasmi Rikmasari* \\ *Department of Prosthodontics, Faculty of Dentistry Universitas Padjadjaran, Indonesia
}

\begin{abstract}
Introduction: Among the most complex restorative challenges for a dentist is that related to determine the occlusal vertical dimension (OVD). Objective methods of determining the OVD offers significant advantages because no radiographs or other special measuring devices are required. Previous review said that the OVD is similar to the pupil-rima oris distance among the population of India. The purpose of this study was to compare whether there was differences between the OVD and the pupil-rima oris distance among Sundanese population. Method: This study was an analytical cross sectional approach. A total of fifty Sundanese students of Faculty of Dentistry, Universitas Padjadjaran who have fulfilled all criteria were chosen randomly to be measured the OVD and the pupil-rima oris distance. Result: Results showed that there was no significant difference between the OVD and the pupil-rima oris distance $(a=5 \%)$. There were strong correlations between the OVD and the pupil-rima oris distance $(p<0.05)$. An analysed with Spearman's rank correlation statistic $(\alpha=5 \%)$ reveals that there was a significant difference between males and females in the OVD and the pupil-rima oris distance. Conclusion: Based on the analysed data, we can conclude that there wass no significant difference between the OVD and the pupil-rima oris distance, thus, the pupil-rima oris distance can be suggested as an objective method to determine the length of OVD in Sundanese population.
\end{abstract}

Keywords: Occlusal vertical dimension, pupil-rima oris, Sundanese population

P-ISSN 1979-0201, e-ISSN 2549-6212 Available from:http://jurnal.unpad.ac.id/pjd/index

DOI:http://dx.doi.org/10.24198/pjd.vol29no2.13657

Submission: Feb 2017 Publishing: July 2017

\section{INTRODUCTION}

The facial growth is an important factor to be considered in Prosthodontics. Complete denture prosthodontics involves the replacement of the lost natural dentition and the associated structures of the maxilla and mandible for the patients with loss of their remaining natural teeth or are soon to lose them. ${ }^{1}$ They are composed of the jaws and teeth, the muscles of mastication, and the temporomandibular system. By being edentulous, the teeth and their associated periodontal ligament nerves are lost, and replacing the function of the lost teeth is the aim of prosthodontics treatment. ${ }^{2}$

One major concern in treating edentulous patient is establishing the correct vertical

Corresponding author: Nur Emalina, Department of Prosthodontics, Faculty of Dentistry Universitas Padjadjaran, Indonesia Jl. Sekeloa selatan No.1 Bandung, Jawa barat. Email: emmalynna94@gmail.com 
dimension of the edentulous mouth. Other than functional importance, the lower third of the face affects one's facial expressions and appearance. Appropriate vertical dimensions, stable occlusal contacts that are harmonious with the existing temporomandibular joints and masticatory muscles, and consistent contours with the surrounding facial musculature will help to adapt the complete dentures to the rest of the masticatory system. ${ }^{2}$

There are several methods in determining the vertical relation of the jaw by using anthropometric landmarks. Previously, Law ${ }^{3}$ carried a study on comparison between the length of OVD with twice the intercanthal distance in adult Mongoloid race in 2011. While in 2010, $\mathrm{Goh}^{4}$ carried a study on the comparison between the length of OVD and the length of thumb.

Despite conflicting evidence in the literature regarding the measuring of the vertical dimension in edentulous patients, the use of facial reference points is still a popular method in clinical practice, and both caliper and the Willis gauge techniques are used in research studies. ${ }^{5}$ Correlation studies between the anthropometric landmarks (pupilrima oris) distance with vertical dimension (subnasion-gnathion) can help the clinicians to establish the correct vertical dimension in the treatment of patients requiring restorations like the complete denture. ${ }^{1}$

The study is carried to evaluate the reliability of facial measurements for determination of OVD in edentulous subjects using accepted facial dimensions recorded from dentulous subjects. Measuring the distance between soft tissue landmarks on the face is one of many other techniques used for predicting the OVD in complete denture restoration. This method is based on the harmony of the face and facial proportions that have been advocated to be relatively constant and unchanged with age progress. ${ }^{6}$

The anthropometry method presents many advantages to the evaluation of craniofacial morphology by using simple, noninvasive, low-risk, and inexpensive techniques. ${ }^{7}$ Therefore, author was interested to study whether the OVD have correlations with the pupil-rima oris distance among the Sundanese population who are represented by the undergraduate Sundanese students of Faculty of Dentistry in Universitas Padjadjaran.

\section{METHODS}

The design of this study was descriptive analytical method and random sampling whereby each sample was observed only one time and the independent and dependent variables were observed and measured simultaneously during that one time of observation to study the relationship between two variables. This study took a survey method with analytical cross sectional approach of the undergraduate Sundanese students batch 2013, 2014 and 2015 of Faculty of Dentistry in Universitas Padjadjaran. Subject's ethnicity was confirmed both by self-identification and others' recognition. ${ }^{8}$ Sunda sub race must be inherited in three generations without interference from any other sub race(s). ${ }^{9}$

The anthropometric landmarks (pupil-rima oris) distance and length of OVD (subnasalegnathion) were measured on each sample and the mean values (using t-test), standard deviation and range were calculated. Spearman's rank correlation coefficient method will be used to test if there is correlation between the mean length of vertical dimension and the pupil-rima oris distance. ${ }^{10}$

The study was carried among male and female samples who fulfills the criteria as stated below. Inclusive criteria from the sample were: age 19-25 years old; a full dentition set (except third molars); class I jaw relation, and a harmonious face; and agree to take part in the research study (informed consent). Exclusive criteria from the sample were: a had missing teeth; malocclusion (open bite, deep bite); class II or III jaw relation; previous or current orthodontic treatment; previous major stomatognathic or plastic surgery; disfigurement of the face; and temporomandibular joint disorders.

Research procedure: Patient with inclusive criteria is given informed consent to be filled; Mouth mirror and digital vernier caliper sterile was used; Patients will be sitting in an upright position, looking forward, head without any support and occlusal plane parallel to the floor; Soft tissue points of pupil on closed eyelids, rima oris, subnasion, gnathion are palpated and then marked on the face with an indelible pencil; To record OVD, subjects are instructed to bite lightly on the posterior teeth in centric occlusion. For centric occlusion, the subjects are asked to bring 
their teeth into contact with their lips relaxed. ${ }^{6}$ The lower tip of the caliper is placed firmly below the chin at gnathion. The upper tip of the caliper then raised until it lightly touched the base of nasal septum; For the second measurement, the pupilrima oris distance is obtained between the center of the pupil and a line projected laterally from the resting median line between the upper and lower lips is measured. Subjects are first asked to focus at a point which was about six feet distance in front of them. This allows the individual eyes to be aligned in the center. The patients are then instructed to close the eyelids without changing

Table 1. Samples based on gender

\begin{tabular}{lll}
\hline Gender & Frequency & Percentage \\
\hline Male & 10 & $20 \%$ \\
Female & 40 & $80 \%$ \\
\hline Total & 50 & $100 \%$ \\
\hline
\end{tabular}

Table 2. Samples based on batch

\begin{tabular}{ccc}
\hline Batch & Frequency & Percentage \\
\hline 2013 & 13 & $26 \%$ \\
2014 & 17 & $34 \%$ \\
2015 & 20 & $40 \%$ \\
\hline Total & 50 & $100 \%$ \\
\hline
\end{tabular}

Table 3. Samples based on age

\begin{tabular}{ccc}
\hline Age & Frequency & Percentage \\
\hline 19 years old & 16 & $32 \%$ \\
20 years old & 13 & $26 \%$ \\
21 years old & 15 & $30 \%$ \\
22 years old & 6 & $12 \%$ \\
\hline Total & 50 & $100 \%$ \\
\hline
\end{tabular}

the position of the eye. To verify that the patient was able to carry such an exercise without error, the patients were asked to open the eyelids again. This would verify the correct position of the eyes. The point of greatest convexity over the closed upper eyelid was considered to be the center of the pupil. The lower tip of the caliper is placed at a line projected laterally from the resting median line between the upper and lower lips. The upper tip of the caliper then raised until it lightly touched the center of the pupil on closed eyelid. ${ }^{5}$ For all the parameters of study mean values (using t-test), standard deviation and range is calculated and recorded and correlation is then studied using Spearman's rank correlation coefficient method. ${ }^{10}$

\section{RESULT}

The result of the research was presented below. The samples were indentified by gender, batch, and age (Table 1-3). There was a correlation between OVD and pupil-rima oris distance for male as well as for female (Spearman's rank correlation was 0.697 for male, 0.688 for female, 0.789 for all samples). So it can be conclude that there was a correlation between OVD with pupilrima oris distance.

\section{DISCUSSION}

The total of samples conducted in this research were $10(20 \%)$ male students and $40(80 \%)$ female students (Table 1 ). Only 10 male samples are involved compared to the 40 female respondents. This is because the total number of male students of Faculty of Dentistry, Universitas Padjadjaran

Table 4. Descriptive of Occlusal Vertical Dimension

\begin{tabular}{ccccccc}
\hline Variable & Sample (gender) & Number & Min $(\mathrm{mm})$ & Max $(\mathrm{mm})$ & Mean & Standard deviation \\
Occlusal & Male & 10 & 62.54 & 75.03 & 67.51 & 3.78 \\
Vertical & Female & 40 & 48.59 & 70.11 & 60.15 & 4.71 \\
Dimension & All & 50 & 48.59 & 75.03 & 61.62 & 5.39 \\
\hline
\end{tabular}

Table 5. Descriptive of Pupil-Rima Oris Distance

\begin{tabular}{ccccccc}
\hline Variable & Sample (gender) & Number & Min $(\mathrm{mm})$ & Max $(\mathrm{mm})$ & Mean & Standard deviation \\
Pupil & Male & 10 & 61.92 & 73.19 & 67.95 & 3.48 \\
-Rima Oris & Female & 40 & 55.35 & 70.34 & 62.79 & 3.31 \\
\cline { 2 - 7 } Distance & All & 50 & 55.35 & 73.19 & 63.82 & 3.91 \\
\hline
\end{tabular}


is fewer compared to the total number of the female students with the ratio of approximately $1: 6$. Table 2 shows the frequency distribution of respondents based on batch. There were 13 (26\%) students of batch 2013, 17 (34\%) students of batch 2014 and 20 (40\%) students were involved. These students are randomly selected and are chosen based on the inclusive criteria of the research. Only a few respondents are selected because the total number of pure Sundanese students with three generation of pure Sunda which fullfulled all the criterias, were limited.

Table 3 shows the frequency of the respondents based on age. The range of age 19-22 is one of the inclusive criteria of this research. Ricketts et al. showed that lower facial height in adults stayed constant with age. Therefore, the students with age range between 19-22 years old among the students of batch 2013-2015 are chosen. In a study by Kesterke et al. ${ }^{11}$, it is stated that eye and mouth measurements showed a very different pattern of dimorphism with increased age, with no major spike around puberty and lower levels into adulthood relative to other craniofacial regions. Therefore, the adult stage and the pupil is used as one of the anthropometric landmarks of this study. ${ }^{11}$

The data showed in Table 4 and Table 5 which shows the descriptive data of OVD and the descriptive data of pupil-rima oris distance respectively. The results showed there was a significant difference in the mean of OVD and the pupil-rima oris distance between male and female samples. This finding is consistent with a study done by Oladipo et al..$^{12}$ In his research, he revealed that the mean values for all the parameters of facial measuremenrs were significantly higher in male than in female subjects $(p<0.05)$, with exception of the nasal height. The difference could be caused by the facial dimensions that are modifies during growth and development with sex-related characteristics. ${ }^{13}$ Individual variation among the example of male and female includes a range of features. Examples of clear differences are found in the length and width of the nose and mouth, the inclinations of forehead and nose, as well as the overall shape of the face. ${ }^{14}$

When subjected to Spearman's Rank Correlation test there is a variation of results in female samples. This may be because within female, there is a layer of subcutaneous fat develops that causes the rounding and softening of contours of the face and body. Whereas, the male subcutaneous fat development is much less pronounced due to the effects of testosterone which reduces fat by aiding fast metabolism which gives the male a leaner and more angular face than the female. ${ }^{15}$ Even there was a variation in female samples, there was still a correlation between the OVD and the pupil-rima oris distance. May due to this variation, the total correlation coefficient of the OVD and the pupil-rima oris distance was lower than the expectation.

The results showed that there was no significant difference in the mean of OVD and the pupil-rima oris distance as a whole and in both male and female samples. This finding is consistent with the findings of Misch $^{16}$ where he stated that the original OVD is most often similar to at least 12 other dimensions on the face and hands and may be objectively determined in most of the patients. One of the dimensions is the pupil-rima oris distance. Although there were many differences between male and female, the proportion of one body part to another body part in one individual was the same for both sex groups. ${ }^{17}$

Previous study by Nagpal et al. ${ }^{1}$ were done in North India which is mostly represented by Caucasian people who belong to the Caucasoid race. According to Nagpal et al. ${ }^{1}$, the OVD is most often similar to the length of pupil-rima oris. On the contrary, this research was conducted among the undergraduate Sundanese students of Faculty of Dentistry Universitas Padjadjaran who belong to Deutero Malay sub-race. This research prove that even in Deutero Malay study samples there was a correlation between the mean length of OVD and mean length of the pupil-rima oris which is similar to Nagpal et al. ${ }^{1}$

Regardless of racial differences and sexual dimorphism between males and females, a proportional relationship exists between the length of OVD and the length of pupil-rima oris. Thus, the length of pupil-rima oris can be suggested to be used as one of the guide in the determination of OVD. Measurement of the length of pupil-rima oris is objective measurements rather than the subjective criteria which requires no radiographs or other special measuring devices and provides reproducible values for future 
reference. This method is attractive and practical because it is simple, non-invasive, reliable, lowrisk and inexpensive techniques. Also, it does not require a great amount of time and experience to master which is another advantage over previous methods. ${ }^{18}$

Besides the pupil-rima oris, there are many other methods in previous studies which is using the anthropometric landmarks to compare with the OVD. Anthropometric measurements were used in order to determine proportions of body parts since antiquity, when sculptors and mathematicians followed the golden proportions, later specified as a ratio of 1.618:1. In line with these observations, this study was carried and designed to assess the possibility of any correlation between the OVD and the length of pupil-rima oris among the Sundanese population so that it can serve as a simple and precise method for estimating OVD. This feature of human anthropometry seems to remain an unturned stone in the field of dentistry. The hypothesis of this research was that there should be a significant relationship between the occlusal vertical dimension and the length of pupil-rima oris. ${ }^{18}$

In a book "Anatomical Studies" by Leonardo Da Vinci contributed several observations and drawings on facial proportions. He found chin-nose distance equal to the superior surface of right ear to inferior surface of right ear, hair line to right eyebrow line and two times of outer canthus of right eye to inner canthus of right eye. In a study by Majeed et al. ${ }^{19}$ which is carried out among 300 Pakistani population, the chin-nose distance mean value was $62.70 \mathrm{~mm}$ while the mean values of the superior surface of right ear to inferior surface of right ear were $59.58 \mathrm{~mm}$ and $58.35 \mathrm{~mm}$ for the mean values of the hair line to right eyebrow line which indicates no close approximation with the chin nose distance. The mean value of two times of outer canthus of right eye to inner canthus of right eye was $63.58 \mathrm{~mm}$ and this value is more significantly related to the chin-nose distance of the study which can be recommended for determination of occlusal vertical dimension. ${ }^{19}$ The findings of Leonardo was agreed by Misch. ${ }^{16}$

$\mathrm{McGee}^{20}$ determined the vertical dimension of occlusion with bridge of the nose to base of the right ala of the nose, right corner of lips to left corner of lips along the curvature, outer canthus of right eye to right angle of the mouth, centre of pupil of right eye to lower border of upper lip and center of pupil of right eye to center pupil of left eye. Nagpal et al. ${ }^{1}$ stated the outer canthus of right eye to right angle of the mouth distance is comparable to chin-nose distance in Indian population. ${ }^{19}$

In the study by Majeed et al. ${ }^{19}$, the mean values of the bridge of the nose to base of the right ala of the nose was $58.51 \mathrm{~mm}$, outer canthus of right eye to right angle of mouth was 67.35 $\mathrm{mm}$, center of pupil of right eye to lower border of upper lip was $65.70 \mathrm{~mm}$, center of pupil of right eye to center of pupil of left eye was 61.41 $\mathrm{mm}$ which did not constitute close approximation for the determination of OVD among Pakistani population. The mean value of right corner of lips to left corner of lips along the curvature more in Caucasians, described by $\mathrm{McGee}^{20}$ gave us mean value of $63.71 \mathrm{~mm}$ which is correlated with the chin-nose distance. ${ }^{19}$

Misch $^{16}$ stated that the OVD is related to twelve different facial measurements which he adopted nine parameters previously described by Ruchi Ladda, Knebelman, Leonardo, McGee and Willis. In addition to those stated, he mentioned three parameters by himself which were right eyebrow line to base of the right ala nose, two times of outer canthus of right eye to inner canthus of right eye and two times of inner canthus of right eye to inner canthus of left eye. ${ }^{16}$

In a study by Majeed et al. ${ }^{19}$ the mean values of the parameters of right eyebrow line to base of the right ala nose was $66.66 \mathrm{~mm}$, the mean values for two times of outer canthus of right eye to inner canthus to right eye was 63.90 and two times of inner canthus of right eye to inner canthus of left eye was $62.29 \mathrm{~mm}$. The result of parameters right eyebrow line to base of the right ala nose and two times of outer canthus of right eye to inner canthus of right eye did not correlate, while the mean value of parameter two times of inner canthus of right eye to inner canthus of left eye coincides with the chin-nose distance. Therefore, the parameter two times of inner canthus of right eye to inner canthus of left eye can be proposed to be used for the determination of OVD among Pakistani individuals. ${ }^{19}$

In a study carried by Basnet et al. ${ }^{2}$ the correlation between the facial measurements 
and occlusal vertical dimension was found statistically significant in both ethnicities of Aryan and Mongoloids. The correlation between the rima oris to pupil distance was stronger than the others. Overall, the distance of rima oris to pupil was significantly correlated to occlusal vertical dimension with stronger correlation than other facial measurements. The result is in contrast with the study done by Delic et al. ${ }^{21}$, where the correlation of eye-ear distance $(r=0.8676)$ was stronger than pupil to rima oris distance $(r=0.4357)$ in their study. In a study done in Phillipinos by Tina-Olaivar et al. ${ }^{22}$ the rima-oris to pupil distance was longer than lower facial height by $3 \mathrm{~cm}$.

The traditional methods such as the judgment of facial esthetics and patient comfort sounds well subjectively but are too nonspecific scientifically. Aids, including tooth display, lip support, harmonious relationship and facial picture do not substantiate for those patients in whom no factual records exists. The objective method like electromyography and biting forces are impractical as they necessitate the use of complex devices and cannot be used routinely. ${ }^{5}$

The facial landmarks can be used reliably to determine occlusal vertical dimension in edentulous patients since they are accessible and relatively unchanged throughout life. If no records are available, one cannot even determine a starting point, the position of mandible would occupy to restore occlusal vertical dimension. Therefore, to overcome these difficulties, an investigation was undertaken to find a simple yet feasible method by studying the relationship between vertical dimension of occlusion and length of craniofacial landmarks, taking into account that the growth of body parts place in proportion to each other. ${ }^{19}$

This study is carried out in order to find the correlation between the occlusal vertical dimension and pupil-rima oris distance and whether the pupil-rima oris distance can be used as a method in determining the OVD which is one of the important factors in prosthodontics department. Loss of teeth results in poor appearance of lower third of the face. Prosthodontist have been seeking for universally accepted method of determining the OVD. There are many methods such as the use of anterior teeth measurements, closest speaking space, swallowing method, patient's neuromuscular perception, cephalometric radiographs, intra-oral and extraoral anatomic landmarks and measurements of fingers. All of the methods possess some kinds of disadvantages. The present research gave some idea about facial measurements and their use in determining occlusal vertical dimension. ${ }^{2}$

Prosthodontics as a whole has progressed leaps and bounds with variety of techniques being proposed and practiced for the evaluation of OVD. However, none of the techniques is scientifically proven more accurate than the other. Each method has its own limitations, they are either tedious, time consuming, require special instrument/equipment, or expose patients to radiation. Furthermore, radiographic set up in order to provide lateral cephalographs or electromyographic machine may not be available in most of the dental offices. ${ }^{18}$

Reestablishing the occlusal vertical dimension not only creates a space between the upper and lower ridges to be occupied by the artificial teeth but also improves the function of the denture prosthesis as well as the esthetic appearance of the patient. Restoring this important component should not involve facial strain or patient discomfort and should be in harmony with the facial dimensions. The greatest danger in the phase of denture construction is an excessive inter-arch distance because premature striking of teeth causes recurring trauma on the tissues and longer leverage, making the denture more awkward to manipulate and displaced easily. This defective occlusal contact may result in clicking of the dentures. ${ }^{23}$

However, a reduced inter-arch distance results in facial expression that is not desirable, and the vertical dimension of the face should be increased to a point that will be satisfactory and comfortable. With a reduced inter-arch distance, the lower third of the face is changed because the chin has the appearance of being too close to the nose. The lips lose their fullness, and the vermillion borders of the lips are reduced to approximate a line. The corners of the mouth turn down because the orbicularis oris and its attaching muscles are pushed too close to their origin. The reduced vertical relation of occlusion reduces the action of the muscles, with resultant loss of muscle tone which gives the face an appearance of flabbiness instead of firmness. ${ }^{23}$ 
A reduced inter-arch distance often results in a disease known as perleche. Reduced interarch distance results in the loss of the cubicle space of the oral cavity. Normally the tongue at rest completely fills the oral cavity, therefore, a reduced inter-arch distance will have a tendency to push the tongue toward the throat, with the result that adjacent tissues will be displaced and encroached upon. Encroachment may mean closure or occlusion of the opening of the Eustachian tube, which would interfere with the function of the ear. ${ }^{5}$

The absence of any pre-extraction record for the occlusal vertical dimension makes the measurement of the vertical height of the lower third of the face arbitrary. However, there are no superior method to establish the original occlusal vertical dimension for all individuals. Therefore, suggestions have been made to use the facial proportions as alternative methods to predict the missing OVD. ${ }^{1}$

The variations in all the measurements found may be due to the differences in measuring techniques, ethnicities of the population and sample size studied. Nevertheless, the results indicated that anthropometric measurements like pupil-rima oris lengths can serve as a basic guide in estimating the lower facial height and offer significant prosthetic advantages. As these are objective measurements rather than subjective criteria's such as resting jaw position or swallowing, the guesswork in OVD is eliminated. Moreover, the OVD estimated using this method is within the range of 2-4 mm which is significantly less if compared to other methods where a range of $0-14 \mathrm{~mm}$ is given. ${ }^{18}$

The limitation of this study was that it was restricted to the subjects with Class I malocclusion and other skeletal or dental malocclusions were not considered. Also, the subjects were not categorized based on facial forms. Further, the measurements is difficult to record when patient has a round facial profile with excessive soft tissue bulk under the chin. ${ }^{18}$

To authenticate these findings, further studies should be carried out comprises of a board clinical research program that would include the similar analysis for dentulous population in other ethnic groups and then appropriate regression equations may be constructed which can be accepted universally. However, the operator should aware that OVD is the result of a mucoskeletal balance. The correct OVD can be described well as a range instead of as a fixed point. Therefore, in order to evaluate the OVD, a pluralistic method should be adopted at all the stages of rehabilitation in order to maximize the benefits and minimize damage to the stomatognathic system. ${ }^{18}$

The results obtained in this research support almost all the hypothesis, hence the length of pupil-rima oris can be suggested to be used as one of the guide in the determination of OVD. However, other method of determination of OVD should be used together in order to obtain a more accurate measurement.

\section{CONCLUSION}

It can be concluded that there was correlation between the OVD and the pupil-rima oris distance among Sundanese population. The length of pupil-rima oris can be recommended to be used as one of the guide in the determination of OVD among Sundanese population when the OVD is already lost.

\section{REFERENCES}

1. Nagpal A, Parkash H, Bhargava A. Reliability of different facial measurements for determination of vertical dimension of occlusion in edentulous using accepted facial dimensions recorded from dentulous subjects. J Indian Prosthod Soc 2014;14(3):233-24.

2. Basnet BB, Parajuli PK, Singh RK, Suwal P, Shrestha P, Baral D. An anthropometric study to evaluate the correlation between the occlusal vertical dimension and length of the thumb. Clinical, Cosmetis and Investigational Dentistry 2015;7:33-9.

3. Law KY. Comparison between the length of vertical dimension of occlusion with twice the intercanthal distance in adult Mongoloid Race (Unpublished minor thesis). Bandung: Universitas Padjadjaran; 2011. p. 2.

4. Goh LT. The comparison between the length of vertical dimension of occlusion and the length of thumb (Unpublished minor thesis). Bandung: Universitas Padjadjaran; 2010. p. 5

5. Brar A, Mattoo KA, Singh $Y$, Singh $M$, 
Khurana PRS, Singh M. Clinical reliability of different measurements in determining vertical dimension of occlusion in dentulous and edentulous subjects. Int J Prosthodon Restorative Dentistry 2014;4(2):68-77.

6. Alhajj $M N$, Khalifa N, Amran A. Eye-rima oris distance and its relation to the vertical dimension of occlusion measured by two methods: anthropometric study in a sample of yemeni dental students. Eur J Dentistry 2016;10(1):29-33.

7. Cattoni DM, Fernandes FD, Di FRC, De LMR. Quantitative evaluation of the orofacial morphology: anthropometric measurements in healthy and mouth-breathing children. Int J Orofac Myol 2009;35(1):44-54.

8. Candramila W, Sumarsono SH, Suryobroto $B$, Moeis MR. Face shape variation among Sundanese people from Western Java, Indonesia. Hayati J Bioscie 2015;22(1):34-40.

9. Prasetyono $\mathrm{TOH}$, Moegni KF. Morphometry of Deutero Malay female nose. Division of Plastic Surgery 2009;18(3):189-92.

10. Nazir S, Zargar NM, Khurshaid SZ, Shah AF, Naz F, Malik M. Correlation between vertical dimension of occlusion and finger length in Kashmiri population. J Orofac Res 2015;5(2):37-9.

11. Kesterke MJ, Raffensperger ZD, Heike CL, Cunningham ML, Hecht JT, Kau CH et al. Using the $3 \mathrm{D}$ facial norms database to investigate craniofacial sexual dimorphism in healthy children, adolescents, and adults. BioMed Central 2016;7(23):1-14.

12. Oladipo GS, Esomonu C, Osogba IG. Craniofacial dimensions of ljaw children and adolescents in Nigeria. J Biomed Int 2010;1(1):25-9.

13. Ferrario VF, Dellavia C, Zanotti G, Sforza C. Soft-tissue facial anthropometry in down syndrome subjects. J Craniofac Surg 2004;15:528-32.

14. DeCarlo D, Metazas D, Stone M. An anthropometric face model using variation techniques. Proceedings of the $25^{\text {th }}$ Annual Conference on Computer Graphics and Interactive Techniques. New York. July 1998. New York: ACM; 1998. p. 67-74.

15. Lewis C, Turner III, Tschen, Jaime A. Klima MD, Marcela MD. Subcutaneous fat necrosis associated with pancreatic islet cell carcinoma. J Am Dermatopathol 1991;13:526.

16. Misch CE. Contemporary implant dentistry. $3^{\text {rd }}$ ed. St. Louis, Missouri; 2008. p. 236

17. Van Der Beek MCJ, Hocksma JB, PrahlAndersen B. Vertical facial growth and statural growth in girls: a longitudinal comparison. $J$ Eur Orthod 1996;18:549-55.

18. Ladda R, Bhandari AJ, Kasat VO, Angadi GS. A New technique to determine vertical dimension of occlusion from anthropometric measurements of fingers. Indian J Dent Res 2013;24(3):316-20.

19. Majeed MI, Afzal M, Kashif M. Determination of occlusal vertical dimension in a section of Pakistani population using craniofacial measurements. Research Gate 2015;6(1):1-5.

20. McGee G. Use of facial measurements in determining vertical dimension. JADA 1947;35:342-50.

21. Delic Z, Simunovic-Soskic M, Perinic-Grzic R, Vukovojac S, Rajic Z, Kuna T. Evaluation of craniometric methods for determination of vertical dimension of occlusion. J Croation Anthrop Soc 2000;1(24):31-5.

22. Tina-Olaivar EO, Olaivar OK. A Comparative study of the upper and lower vertical facial measurements of the Filipinos as it is used in the willis method for determining the vertical dimension of occlusion. J Phillipp Dent Assoc 1998;50(1):44-8.

23. Boucher CO. Boucher's prosthodontic treatment for edentulous patients. Great Britain: Mosby; 1997. p. 118-20. 